\title{
Favola, mito ed altri saggi di letteratura e filologia in onore di Gianni Mombello, a cura di Antonella Amatuzzi e Paola Cifarelli
}

\section{Cristina Musio}

\author{
(2) OpenEdition \\ Journals \\ Edizione digitale \\ URL: https://journals.openedition.org/studifrancesi/26198 \\ DOI: $10.4000 /$ studifrancesi.26198 \\ ISSN: 2421-5856

\section{Editore} \\ Rosenberg \& Sellier

\section{Edizione cartacea} \\ Data di pubblicazione: 1 avril 2007 \\ Paginazione: 171-172 \\ ISSN: 0039-2944

\section{Notizia bibliografica digitale} \\ Cristina Musio, «Favola, mito ed altri saggi di letteratura e filologia in onore di Gianni Mombello, a cura di \\ Antonella Amatuzzi e Paola Cifarelli», Studi Francesi [Online], 151 (LI | I) | 2007, online dal 30 novembre \\ 2015, consultato il 23 novembre 2021. URL: http://journals.openedition.org/studifrancesi/26198; DOI: \\ https://doi.org/10.4000/studifrancesi.26198
}

Questo documento è stato generato automaticamente il 23 novembre 2021.

\section{cc) (†)}

Studi Francesi è distribuita con Licenza Creative Commons Attribuzione - Non commerciale - Non opere derivate 4.0 Internazionale. 


\title{
Favola, mito ed altri saggi di letteratura e filologia in onore di Gianni Mombello, a cura di Antonella Amatuzzi e Paola Cifarelli
}

\author{
Cristina Musio
}

\section{NOTIZIA}

Favola, mito ed altri saggi di letteratura e filologia in onore di Gianni Mombello, a cura di Antonella AMATUZZI e Paola CIFARELLI, presentazione di Lionello Sozzi, «Franco-Italica», 23-24, 2003, pp. XXXII-618.

1 Il volume comprende una trentina di saggi, suddivisi in tre sezioni: «Entre fable et mythe, L'Art d'écrire au Moyen Age et au XvI ${ }^{\mathrm{e}}$ siècle» e «Portraits réels et imaginaires». Qui ci occuperemo soltanto dei contributi relativi al XVII secolo, per gli altri periodi si vedano le sezioni corrispondenti (a partire dal nº 146 della rivista).

Michele MASTROIANNI, Tra favola ed emblema: i 'Sonnets franc-comtois' attribuiti a Chassignet, pp. 37-83. Dopo un excursus sugli studi riguardanti l'emblematica e la favolistica di area francese, Mastroianni si concentra su di «un'opera charnière fra Manierismo e Barocco»: i Sonnets Franc-Comtois, raccolta attribuita a Jean-Baptiste Chassignet e composta verso il 1615. La struttura di ciascun componimento è quadripartita: motto, immagine, epigramma (due ottosillabi a rima baciata) e sonetto; questi due ultimi elementi rappresentano la spiegazione dei primi due. L'A. fa un raffronto con lo schema esemplare della forma-emblema di Alciato (1531) e Corrozet (1544) e si concentra poi sugli elementi favolistici e quelli di natura religiosa presenti nella raccolta.

Cecilia RIZZA, Entre mythe et fable. Petite histoire des deux mots au XVII siècle, pp. 111-123. La parola "mythe" nel xVII secolo non appartiene ancora al linguaggio corrente e neppure a quello letterario, infatti è totalmente assente sia dai principali dizionari dell'epoca 
che dai testi di quei poeti e drammaturghi che del mito hanno fatto largo uso. Al contrario, la parola "fable" ricorre molto spesso e l'A. ne analizza le varie accezioni citando molti esempi da dizionari e da testi letterari, nei quali "fable" spesso è sinonimo di "mythe", nel sigificato moderno della parola. Ma "fable" è un vocabolo polisemico e nei testi del XVII secolo spesso ricorre anche con connotazione negativa, nel senso di "fausseté" e "risée". Bisognerà aspettare ancora molti anni perché "mythe" entri a far parte della lingua francese e acquisisca le connotazioni e i significati moderni.

4 Lionello sozzi, Les compagnons d'Ulysse, pp. 125-137. Dal confronto tra la favola Les compagnons d'Ulysse di La Fontaine (XII, 1) con tre fonti a lui probabilmente note (il dialogo "morale" di Plutarco nella traduzione di Amyot, l'Asino d'oro di Machiavelli e il dialogo di Giovan-Battista Gelli intitolato La Circe), l'A. conclude che il favolista francese si distacca dai modelli citati in quanto evita i tradizionali argomenti volti ad esaltare la condizione umana ed indirizza la sua ironia tanto verso gli animali quanto verso gli uomini. Oltre ad analizzare i rapporti tra la favola francese e le sue fonti, Sozzi ricava anche nuovi significati dal discorso di La Fontaine: si tratta di un testo che si rivolge al figlio del Dauphin, in un momento che vede la Francia impegnata in una campagna militare considerata forse come troppo "temeraria" e l'A. ne estrapola «una lezione rivolta ai potenti perché saggiamente ritornino sui sentieri fioriti di una pacifica concordia».

5 Gabriella Bosco, La tarasca e il diavolo, pp. 139-154. Dopo un excursus sulle varie apparizioni in letteratura della 'Tarasca', essere fantastico, variamente rappresentato nella tradizione dapprima pagana e poi cristiana come figura mostruosa e terrificante, l'A. si concentra sulle trasposizioni poetiche di età barocca nelle quali è presente questo mostro mitologico, e in particolare sulla Magdaliade ou Esguillon Spirituel, del frate certosino Marc-Antoine Durant (pubblicato a Tours nel 1622). Si tratta di un poema in cinque canti sulla vita e le opere di Maria Maddalena. Nell'ultimo canto viene narrato l'episodio della vittoria di Marta, sorella di Maria Maddalena, sulla Tarasca, la cui descrizione è accostabile a quella del «gran nemico de l'umane genti» nel quarto canto della Gerusalemme Liberata. Dopo il confronto con il Tasso e risalendo all'indietro alle fonti utilizzate dai due autori, Bosco conclude l'ampio percorso effettuato dal mostro attraverso le tradizioni pagana e cristiana.

Daniela DALLA VALLE, La comparsa dei corsari nella letteratura francese del Seicento. Le novelle di Charles Sorel, pp. 155-169. La prima apparizione del "corsaire" nella letteratura francese sembra avvenire in due novelle di Sorel, Le pauvre généreux e Les mal mariés (in Nouvelles Françaises del 1623), rispettivamente con un "Corsaire Turc" e un "Corsaire Ecossais". Prima di analizzare questi testi l'A. delinea un ritratto della figura del corsaro, riassumendo i tratti salienti che lo contraddistinguono nella cultura moderna. Dallo studio dei due personaggi in Sorel emergono già le caratteristiche essenziali del "mito" del pirata/corsaro, anche se quest'ultimo è per il momento solo un personaggio subalterno che si contrappone al protagonista della novella, e questo fatto accentua la doppia dimensione di valenze positive e negative che in seguito saranno incarnate da un solo personaggio, il corsaro appunto, che diventerà così il protagonista della vicenda.

7 Simona mUnARI, Tasso e Petrarca nel romanzo moresco francese del Seicento, pp. 171-190. Il contributo, corredato di bibliografia, propone in primo luogo un'analisi di Mathilde, lunga novella (1667) di Mlle de Scudéry ascrivibile in parte al genere moresco e nella 
quale appaiono le figure di Laura e Petrarca in veste di coprotagonisti. In seguito, attraverso vari esempi, indaga l'influenza della letteratura italiana sull'espressione dell'atmosfera moresca secondo l'interpretazione francese, in particolare nell'Histoire de Célimaure et de Félismène (1665) di Jean Le Rou.

Antonella AMATUZzI, Etude linguistique de la correspondance de René Favre de la Valbonne avec la Cour de Turin, pp. 441-461. Il contributo propone una minuziosa analisi linguistica (dal punto di vista ortografico, fonetico, morfosintattico, lessicale e stilistico) della corrispondenza di René Favre, seigneur de la Valbonne, con La Corte di Torino tra il 1620 e il 1656. Il carteggio è relativo alla controversia scaturita in seguito alla pubblicazione di Le bien public pour le fait de la justice di Favre, opera dal tono spesso critico nei confronti del sistema giudiziario dell'epoca. Dal punto di vista linguistico, questa corrisponedenza costituisce una preziosa testimonianza in quanto riflette lo stato della lingua francese in un periodo in cui si sentiva fortemente la necessità di stabilire delle regole precise.

9 Emanuele KANCEFF, Le "coppole chiatte": antiche immagini francofone dell'Abruzzo, pp. 463-473. Il saggio presenta due resoconti di viaggio del XVII secolo: il primo è Voyage dans le Royaume de Naples del libertino Jean-Jacques Bouchard, mentre il secondo è del geografo Pierre Bergeron. Questi testi dimostrano come, già nella prima metà del 600, l'Abruzzo facesse parte dei percorsi in Italia dei viaggiatori stranieri.

Giorgia PUTTERO, Un nipote del cardinal Mazzarino: Filippo Mancini, «ni courtisan, ni guerrier, ni chrétien», pp. 475-495. Con l'ausilio di svariati documenti dell'epoca, in particolare alcune lettere di Mazzarino, l'A. delinea un "ritratto familiare" di una delle figure più importanti del Seicento francese, concentrandosi perlopiù su «quei nipoti e quelle nipoti che [Mazzarino] fece arrivare alla corte di Parigi in più ondate». Puttero pone l'attenzione in particolar modo sull'arrivo in Francia di Filippo Mancini (figlio della sorella del cardinale), che a causa del suo «spirito libero, artistico e un po' irresponsabile» non fu mai particolarmente apprezzato dallo zio.

11 Ernesto Bellone, Teologi e personale sanitario attivi in Piemonte nel Seicento. Appunti per la storia dell'Università di Torino, pp. 497-519. Questo saggio propone uno studio riguardante la vita dell'Università di Torino nel '600. L'A. riporta il numero di dottori e studenti in teologia e medicina, suddivisi cronologicamente e per area geografica di provenienza, per ridimensionare «quanto si ripete sulla scarsa incidenza dell'Università torinese nella vita sociale del Seicento».

12 Monica PAVESIO, Il viaggio di 'La dama duende' nel XVII secolo: Spagna, Italia e Francia, pp. 521-534. La commedia L'esprit follet (1642) di Antoine Le Métel d'Ouville sembra essere il primo adattamento francese di La dama duende di Pedro Calderón de la Barca (composta probabilmente nel 1629). L'A. passa in rassegna le principali innovazioni apportate dal drammaturgo francese al testo/fonte spagnolo e si concentra in seguito su due testi italiani: La Fantasma overo la Dama spirito (1645) di Teodoro Ameyden e La Dama spirito folletto di Giovan Battista Ricciardi. Il primo testo contiene pochi elementi innovativi e può infatti essere considerato una traduzione fedele de La dama duende; le innovazioni apportate nel secondo testo sono invece notevoli ed accostabili alla versione di d'Ouville. Nella parte finale del contributo Pavesio ipotizza l'influenza della commedia dell'arte su d'Ouville e Ricciardi: quest'ipotesi giustificherebbe le notevoli somiglianze tra i due testi. 